\title{
EVALUATION OF SIGNIFICANCE OF ABSENT NASAL BONE AS ISOLATED RISK FACTOR FOR CHROMOSOMAL ANOMALIES
}

\author{
Dr.M.Ramesh MDRD \\ Assistant Professor Of Radiology, Government Medical College, Omandurar Estate, Chennai 2
}

\begin{abstract}
Background: In India, there is an accelerating demographic switch to non-communicable diseases among which congenital malformations and genetic disorders are important causes of morbidity and mortality. Due to the high birth rate in India, large number of infants with genetic disorders are born every year almost half a million with malformations and 21,000 with Down syndrome and pose a big financial and social burden to nation and family. Hence identifying down syndrome in antenatal ultrasound and karyotyping will help to reduce the incidence of down syndrome and thereby reducing the social and economical constraints.In this study we are testing the importance of isolated absent nasal bone in the diagnosis of trisomies as this was easy to demonstrate by antenatal ultrasound at 11-14 weeks gestation.Comparing the results with nuchal fold thickness and karyotyping with either amniocentesis or chorionic villous sampling .

Methods: The study was conducted in on pregnant mothers who come for NT scan between 11-to 13.6 weeks for our study. Mothers with hypoplastic nasal bone and/or absent nasal bone were further followed with serum markers and later confirmed with karyotyping. Mothers with normal NT scan and nasal bone were further evaluated mainly with serum markers and few with karyotyping who gave consent for it .

Out of 15 cases, 11 cases showed abnormality as follows with 7 cases of both absent nasal bone and increased translucency, 2 cases of isolated nuchal tranlucency and 2 case of isolated absent nasal bone. Out of 15 abnormal cases , 10 patients showed abnormal quadraple test. 7 cases of increased nuchal translucency and absent nasal bone, 2 cases of increased nuchal translucency and 1 case of isolated nasal bone showed high risk for trisomies in quadruple test.
\end{abstract}

Conclusion: Isolated ultrasound finding of absent nasal bone has got insignificant chances of down syndrome and trisomies

\section{INTRODUCTION}

India and other developing nations facing a rising incidence of non cmmunicable diseases Though among the non communicable diseases genetic disorders a rare occurrence, there is a rising trend in genetic disorders especially Down syndrome due to late age of marriage and conception .Due to the high birth rate in India ,a very large number of infants with genetic disorders are born every year about half a million with malformations and 21,000 with Down syndrome. The frequency of Down syndrome among 94,610 births was 0.87 per 1000 , or 1 per 1150 . The children with Down syndrome can develop many complications like heart defects, intestinal abnormalities like Duodenal ateria, Thyroid problems, Leukemia. Hearing loss, Vision problems. Respiratory problems like pulmonary hypertension, Alzheimer's disease .As the result the childrens with Down syndrome pose a big financial and social burden to nation and family. Hence identifying down syndrome in antenatal ultrasound is foremost importance . Karyotyping is gold standard for diagnosis but it is invasive test with a small risk of fetal loss. Nuchal translucency values varies with gestation's age and there is more chance of error in measurement due to subjective variation. Hence in this study we are evaluating the absent nasal bone alone can be significant in diagnosing trisomies , because chances of error is less as there is no measurement and it is non invasive test

1.a Serum markers: The (ACOG) has developed guidelines where women of 35 years and older are typically considered to be at highest risk are offered chorionic villous sampling (CVS) or genetic counseling and amniocentesis, whereas women younger than 35 can be screened using quadruple test with detection rate for Down syndrome to about 80 percent. PAPP-A and HCG measurements are effective for screening only in the first trimester, and alpha-fetoprotein, unconjugated estriol, and inhibin are useful only in the second trimester .In early pregnancy [ 10 weeks] non-invasive maternal serum fetal cell free (cfDNA) is highly sensitive and specific test and reporting also is simple [ very high or very low risk]. serum markers in quadruple test are serum alpha fetoprotein, serum estradiol ,serum B HCG and serum inhibin 
Alpha-Fetoprotein (AFP ) -AFP is initially produced in the fetal yolk sac and liver. A small amount also is produced by the gastrointestinal tract. By the end of the first trimester, nearly all of the AFP is produced by the fetal liver..The AFP concentration in maternal serum rises throughout pregnancy, from a nonpregnancy level of 0.2 to about $250 \mathrm{ng} / \mathrm{mL}$ at 32 weeks gestation.AFP is elevated in open NTD,omphalocele, gastroschisis, congenital renal disease, esophageal atresia, and other fetal distress situations such as threatened abortion, fetal demise and multiple pregnancies. Lower maternal serum AFP values is seen in trisomy 21 (Down syndrome) and trisomy 18.

Estriol (uE3) - The principal estrogen hormone during pregnancy, is synthesized by the intact feto-placental unit.. Estriol levels increase during the course of pregnancy. Decreased unconjugated estriol is a marker for Down syndrome and trisomy 18. Low levelsmay also be seen in pregnancy loss, Smith-Lemli-Opitz, and X-linked ichthyosis (placental sulfatase deficiency). Human Chorionic Gonadotropin- The beta subunit determines the unique physiological, biochemical, and immunological properties of hCG. hCG is synthesized by placental cells in very early in pregnancy, and serves to maintain the corpus luteum, and begins to fall as the role of the corpus luteum in maintaining pregnancy diminishes.Increased levels are associated with Down syndrome, while decreased levels may be seen in trisomy 18 . Elevations of hCG also can be seen in multiple pregnancies, triploidy, fetal loss, and hydrops fetalis.

Inhibin A -secreted by the feto-placental unit during pregnancy. Maternal inhibin A levels is correlated with maternal hCG levels and are abnormal in the same conditions that are associated with abnormal hCG levels (eg, inhibin A levels are typically higher in Down syndrome pregnancies).

Down syndrome - Calculated screen risks $<1 / 270$ are reported as screen negative

screen risks $>$ or $=1 / 270$ are reported as screen positive.

Trisomy 18 - Calculated screen risks $<1 / 100$ are reported as screen negative, screen risks $>$ or $=1 / 100$ are reported as screen positive..

\section{Interpretation}

A negative screen does not guarantee the absence of trisomy 18 or Down syndrome. Screen test has detection rate of approximately $77 \%$ to $81 \%$ of Down syndrome with a false-positive rate of $6 \%$ to $7 \%$. and $60 \%$ to $80 \%$ for trisomy 18 . Screening efficiency increases with maternal age. The estimated risk calculations and screen results are dependent on accurate information for gestation, maternal age, race, IDD, and weight.

bAneuploidy screening-All women, regardless of age, should be offered aneuploidy screening before 20 weeks' gestation .Screening typically provides information about the patient's age-related risk; serum analyte levels; and, if available, nuchal translucency measurements. A numeric risk assessment allows the patient to determine the risk and consequences of giving birth versus proceeding with diagnostic testing.If the screening test is positive and the patient chooses to proceed with a diagnostic procedure (e.g., CVS, amniocentesis), there is a higher chance of discovering an aneuploid fetus than if the woman had not undergone screening. If screening is done, fewer invasive diagnostic procedures would be needed to find a fetus with aneuploidy, and performing fewer diagnostic tests would reduce the number of procedure-related losses of normal fetuses. First trimester screening is combined screening of nuchal translucency measurements, serum markers [PAPP-A and beta-hCG], and maternal age. Second trimester screening involves quadruple screening and maternal age .Genetic counseling and CVS or second-trimester amniocentesis should be offered to women who are found to have an increased risk of aneuploidy during first-trimester screening. Those who are negative for first trimester screening should be offered second trimester quadriple screening. Women should be offered targeted ultrasonography, fetal echocardiography, or both if they have a fetal nuchal translucency measurement of at least $3.5 \mathrm{~mm}$ despite other factors (e.g., negative result on aneuploidy screen, normal fetal chromosomes) because these fetuses are at a significant risk of congenital heart defects, abdominal wall defects, diaphragmatic hernias, and genetic syndromes. Integrated screening is defined as first- and second-trimester screening used to adjust the patient's age-related risk and are reported after both first- and second-trimester tests are done .One trial found that integrated screening using only serum had a detection rate of 85 to 88 percent, however, there is a longer wait time (three to four weeks) between initiation and completion of screening, which may cause increased anxiety for 
some patients..Sequential screening are two types: stepwise and contingent. With stepwise screening, high-risk patients can opt out of continued screening and instead receive genetic counseling and diagnostic testing, and low-risk patients can continue with second-trimester screening. With contingent screening, pregnancy is classified as low, intermediate, or high risk based on first-trimester screening results. Women at high risk are offered CVS, women at intermediate risk are offered continued screening in the second trimester, and women at low risk have no further testing. In theory, contingent-type sequential testing would maintain a higher detection rate while reducing the number of second-trimester screening tests being performed.

\section{1.c Ultrasound markers}

First Trimester -Potential markers for Down syndrome include nonvisualized nasal bone, tricuspid regurgitation, crown-rump length, femur and humeral length, head and trunk volume, and umbilical cord diameter.

\section{Second Trimester - Second-trimester ultrasound markers have low sensitivity and specificity for} detecting Down syndrome,

1.d diagnostic tests. Once when a fetus with absent nasal bone is diagnosed. confirmation with gold standard test of karyotyping by chorionic villous sampling in first trimester or amniocentesis in second trimester is done

Chorionic villous sampling

\section{Indications of CVS -}

1 Abnormal first trimester screen results

2 Increased nuchal translucency

3 other abnormal ultrasound findings

4 family history of chromosomal anomalies

5 Advanced maternal age

Normal result - no signs of any genetic defect

Abnormal result - Downsyndrome ,Hemoglobinopathies, Taysach Disease, Sex Linked

Disorders Risks of the procedure are

Abortion - $1 / 100$ cases

Infection

Spotting and cramping

Rupture of membranes

Limb reduction defects when performed less than 9 weeks

Contraindications

Women with active infection

Carrying monozygotic twins

Amniocentesis

\section{Indications}

1 Diagnostic - abnormal ultrasound,family history, increased maternal age,previous istory of chromosomal anomalies

2 therapeutic - decompression of uterus in unresponsive cases of chronic hydromnios, intrauterine fetal transfusion in severe hemolysis due to rh isoimmunisation, amnioinfusion for oligohydromnios

3 lung maturity by looking for lecithin and spingomyelin ratio in amniotic fluid

\section{complications maternal}

1 cramping

2 preterm labour and delivery

3 PROM

4 Uterine bleeding 
5 maternal infection

6 maternal isoimmunisation

Fetal

1 abortion

2 fetal trauma

3 oligohydromnios

4 respiratory distress

5 club foot

Aim: To evaluate the significance of absent nasal bone in the diagnosis of Downs syndrome and trisomies by considering the karyotyping as gold standard

\section{Review Of Literature}

Recent studies reveal that the nasal bone hypoplasia can be evaluated with sonography and appears as an absence or shortened nasal bone (Kanellopulos 2003, Otano 2002, Orlandi 2003) in prenatal screening. The sonographic identification of this feature has increased the detection of $60 \%$ to $80 \%$ of trisomy fetuses with false positive rate of 5\% (Nyberg 2001, Bromley 2002). The absence of nasal bone in second trimester of pregnancy has a sensitivity of $41 \%$ and specificity of $100 \%$ in detecting fetal Trisomy 21(Anthony Vintzileos 2003). Nasal bone hypoplasia during the second trimester is associated with a high risk of Trisomy 21 and has been suggested as a highly sensitive and specific sonographic marker for chromosomal aneuploidy(Bromley 2002, Cicero 2003, Odibo 2004, Guis Ville 1995).

Cicero et al. 13 found that at the 11-14-week scan the nasal bone was absent in 43 of 59 (73\%) cases of trisomy 21 , while it was absent in only three of $603(0.5 \%)$ chromosomally normal fetuses. The authors found a likelihood ratio of 146.0 for an absent nasal bone. these data are confirmed, the impaired ossification of the fetal nose

Sonek and Nicolaides have recently reported three cases of trisomy 21 in the mid-trimester, in which the nasal bone was absent or shorter than expected for the gestational age. They concluded that the measurement of the fetal nose might improve ability to detect Down syndromee will prove to be one of the most specific markers for trisomy 21. In a study on association between first trimester absence of fetal nasal bone on ultrasound and Down syndrome by, Igarzábal L, Matayoshi T, Gadow EC. showed that absence of nasal bone by first trimester ultrasound was significantly associated with Down syndrome. nasal bone was visible in more than $99 \%$ of karyotypically normal fetuses. 2169 singleton fetus from January 2002 to February 2005, by measuring nasal bone length in 2169 singleton fetuses on a midsagittal plane at 12-14 weeks' gestation.The nasal bone length measurement showed a significant increase with gestational age $(\mathrm{GA})(\mathrm{p}<0.05)$. A linear relationship between nasal bone length and gestational age $(\mathrm{NBL}=-3.3462 \times \mathrm{GA}+0.627, \mathrm{R}(2)=0.30, \mathrm{p}<0.001)$ as well as a linear relationship between nasal bone length and crown-rump length (CRL) (NBL $=0.3741 \mathrm{x}$ $\mathrm{CRL}+0.284, \mathrm{R}(2)=0.30, \mathrm{P}<0.001)$ was established. In a study of Integrated ultrasound and biochemical screening for trisomy 21 using fetal nuchal translucency, absent fetal nasal bone, free beta-hCG and PAPP-A at 11 to 14 weeks.by Bindra R, Rembouskos G, Nicolaides KH,they concluded that integrated sonographic and biochemical test at 11 to 14 weeks can potentially identify about $90 \%$ of trisomy 21 fetuses for a false-positive rate of $0.5 \%$.The nasal bone was absent in $69 \%$ and present in $31 \%$ of the trisomy 21 fetuses suggesting that absent fetal nasal bone alone is $69 \%$ sensitive detection of chromosomal anomalies In a study of first-trimester nuchal translucency, nasal bones,and trisomy 21 in selected and unselected populations .they concluded that First-trimester nasal bones assessment for trisomy 21 screening has a very different performance in selected and unselected pregnancies .The sensitivity, specificity, positive and negative predictive value of absent nasal bones for trisomy 21 were $16.7 \%, 97.3 \%, 1.1 \%, 99.8 \%$ and $47.6 \%, 95.3 \%, 33.3 \%, 97.4 \%$ in the unselected and selected population, respectively. 


\section{Materials And Methods}

The study was conducted in our institution. We selected patients who come for NT scan between 11-to 13.6 weeks for our we used GE voluson ultrasound for our study with curvilinear probe with 3.5 to $5 \mathrm{MZ}$. we included all the antenatal mother referred for nuchal tranlucency scan in our study. Age of the mothers were between 22 and 38 years and the average age is 32 years .mothers were informed about the study. Mothers with hypoplastic nasal bone and/or absent nasal bone were further referred for serum markers like serum PAPPA,B HCG if the mother comes in first trimester and quadruple test if the motehr comes in the second trimester and later confirmed with karyotyping by aspirating aminiotic fluid / chorionic villous sampling. Mothers with normal NT scan and nasal bone were further evaluated with serum markers ,but karyotyping was not done because almost all of them did not give consent for invasive procedure of karyotyping as the chances of losing pregnancy is present in these invasive tests, whereas the mothers with fetus having absent or hypoplastic nasal bone and increased nuchal translucency gave consent as it was the confirmatory test for diagnosis and also to know the trisomy type. In addition of nuchal translucency and nasal bone, other soft markers and major congenital anomalies also screened during the same scan but not considered in our study.

All the antenatal nuchal translucency and nasal bone assessment was done by transabdominal method The gestational period must be 11 to $13+6$ weeks or CRL between $45-84 \mathrm{mms}$. The magnification of the fetus must be such that only the head and upper thorax are present on the screen.A mid sagittal section of the fetal profile is obtained with the ultrasound transducer held parallel to the direction of the nose. The echogenicity of the nasal bone should be greater that the skin overlying it. we usually see 3 lines if the position is correct .The first two lines, which are proximal to the forehead, are horizontal and parallel to each other, resembling an equal sign • . The top line represents the skin and bottom one, which is thicker and more echogenic than the overlying skin, represents the nasal bone. A third line, almost in continuity with the skin, but at a higher level, represents the tip of the nose.Absence of the bottom line of the equals sign represents the absence of the nasal bone. When the nasal bone line appears as a thin line, less echogenic than the overlying skin, it suggests that the nasal bone is not yet ossified, and it is therefore classified as being absent.

In our study we screened for nuchal transluceny as the patient for it only. The following chart to assess the translucency which is based on the standard reference table .

Expected nuchal translucency

\begin{tabular}{|lccc|}
\hline CRL ( & 5m) & th percentile & 50th percentile $^{\text {95 }}{ }^{\text {th }}$ percentile \\
\hline 40 & 0.3 & 1.2 & 2.1 \\
\hline 45 & 0.4 & 1.3 & 2.2 \\
\hline 50 & 0.5 & 1.4 & 2.3 \\
\hline 55 & 0.6 & 1.5 & 2.4 \\
\hline 60 & 0.7 & 1.6 & 2.5 \\
\hline 65 & 0.8 & 1.7 & 2.6 \\
\hline 70 & 0.9 & 1.8 & 2.7 \\
\hline 75 & 0.9 & 1.9 & 2.8 \\
\hline
\end{tabular}

\section{Results}

Out of 1546 studies, 15 cases showed abnormality and 1531 cases were normal sonographically Most of the mothers who showed abnormality were more than 30 years.Out of 15 abnormal sonography, 8 of them showed both absent nasal bone and increased nasal translucency for the given CRL, 3 mothers showed isolated increased nasal translucency with bone, 4 of them showed absent nasal bone with normal nuchal translucency.Amniotic fluid analysis was performed for karyotyping in all 15 of the cases. Out of 15 cases, 11 cases showed abnormality with trisomy 21 in 9 cases and other trisomy in 2 cases .Out of 11 cases ,7 cases are absent nasal bone and increased translucency, 2 cases are isolated nuchal tranlucency and 2 cases are isolated absent nasal bone showed trisomies. 1 case of increased nasal translucency and 
absent nasal bone, 2 cases of isolated absent nasal bone fetuses and 1 of the increased translucency case showed normal karyotyping. Out of 15 abnormal cases , 10 patients showed abnormal quadraple test. 7 cases of increased nuchal translucency and absent nasal bone, 2 cases of increased nuchal translucency and 1 case of isolated nasal bone showed high risk for trisomies in quadruple test .Statistical analysis showed when isolated nuchal tranlucency is considered for diagnosing trisomies by comparing with karyotyping the $\mathrm{P}$ value is 0.564 suggesting that there is no stastistical significance. Though isolated nuchal transluceny patients shows $66 \%$ chance for diagnosing trisomies which is better when compared to patients without any ultrasound finding or with isolated nasal bone in diagnosing trisomies, it is not statistically significant. From the analysis it is clear that the combined ultrasound finding of increased nasal translucency and absent nasal bone shows significant $\mathrm{P}$ value with $87.5 \%$ of cases showing karyotype positivity and indirectly increased chances of trisomies and Down syndrome, whereas isolated ultrasound finding of either increased nasal translucency or absent nasal bone shows insignificant $\mathrm{P}$ value suggesting that though there is difference between these isolated ultasound finding and those without any ultrasound finding, difference is insignificant From the analysis it is clear that the chances of karyotype positivity increases with maternal age

\section{Discussion}

The risk of down syndrome can be calculated based on maternal age, nuchal translucency thickness and blood investigations between 10-13 weeks and 15-20 weeks of gestation .some of the key markers like evaluation of nasalbone, linear arrangement of AV valves, short femur and humerus relative to head size, fetal hydronephrosis, echogenic intracardiac focus.increased nuchal fold thickness alone can diagnose trisomies . it calculates the risk of trisomies .when in addition to increased nuchal fold thickness ,quad test is positive the risk multiplies by 17 times. Non ossified nasal bone and linear arrangement of AV valves increases the risk by 30-60.The incidence of absent nasal bone related to NT,CRL, ethnic origin and aneuploidy.The software first calculates risk based on maternal age,fetal nuchal translucency and maternal serum markers.If the risk is 1 in 50 and if the nasal bone is present, the risk does not change .If the risk is between 1in 50-1000 and if the nasal bone is present, the risk usually reduces and if nasalbone is absent the risk increases.isolated nasalbone absence shows no significant risk. From our study it is clear that isolated nasal bone absence shows insignificant risk and risk is significant if associated with increased nasal translucency

\section{Conclusion}

Combined ultrasound finding of increased nasal translucency and absent nasal bone fetuses have more and significant chances of trisomies and Down syndrome .Isolated ultrasound finding of either increased nasal translucency or absent nasal bone has got insignificant chances of down syndrome and trisomies. Down syndrome and trisomies increases with maternal age is again proved by this study .Fetuses with more increased NT thickness has got more chances of trisomies and Down syndrome

\section{Annexures}

Sonoanatomy

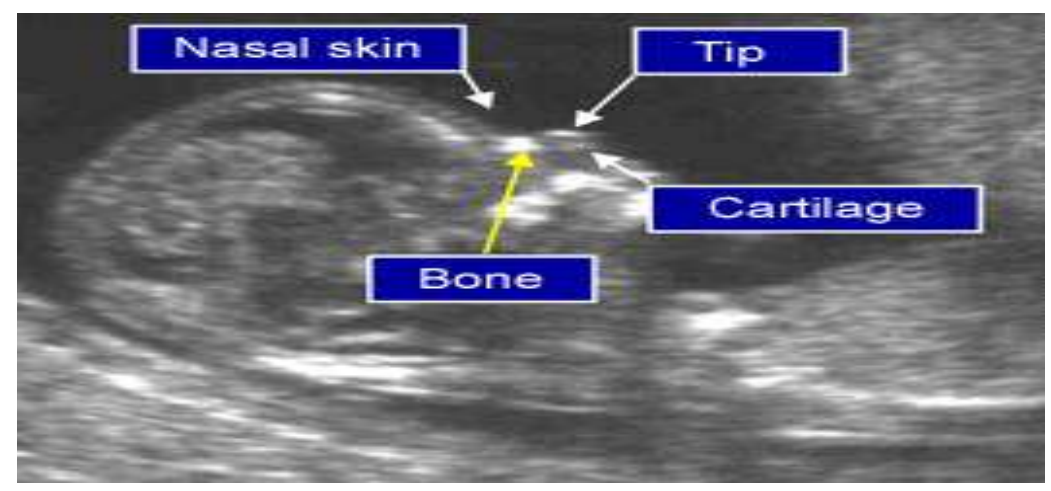


The first two lines, which are proximal to the forehead are horizontal and parallel to each other, resembling an equal sign •. The top line represents the skin and bottom one, which is thicker and more echogenic than the overlying skin, represents the nasal bone.A third line, almost in continuity with the skin, but at a higher level, represents the tip of the nose sample cases

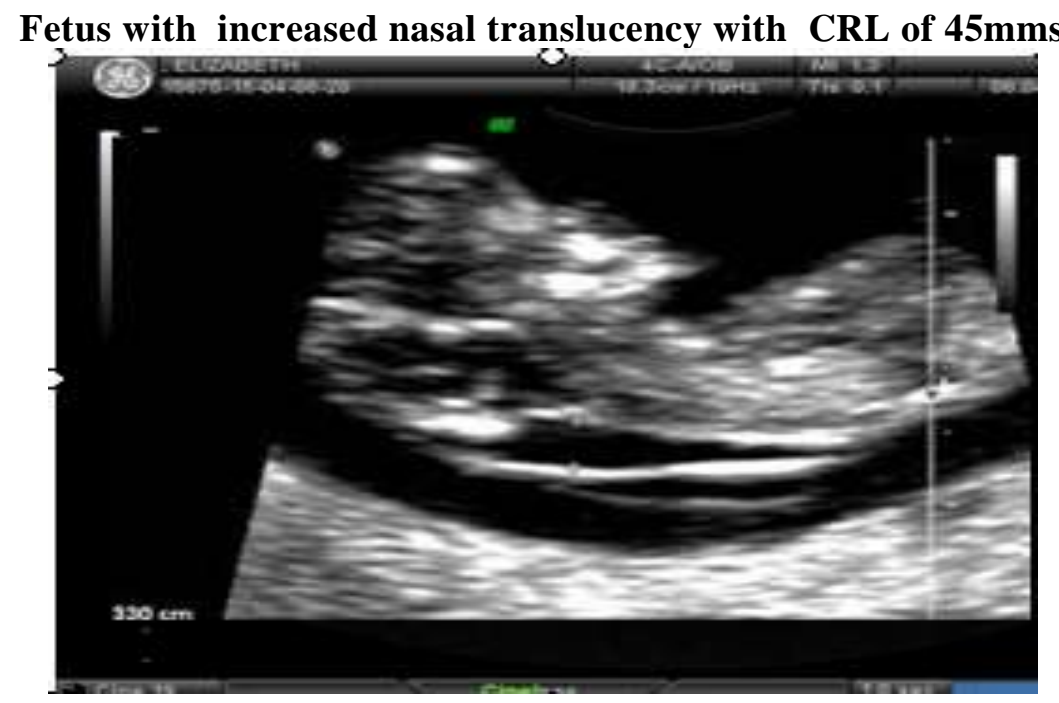

Fetus with absent nasal bone with normal nuchal translucency

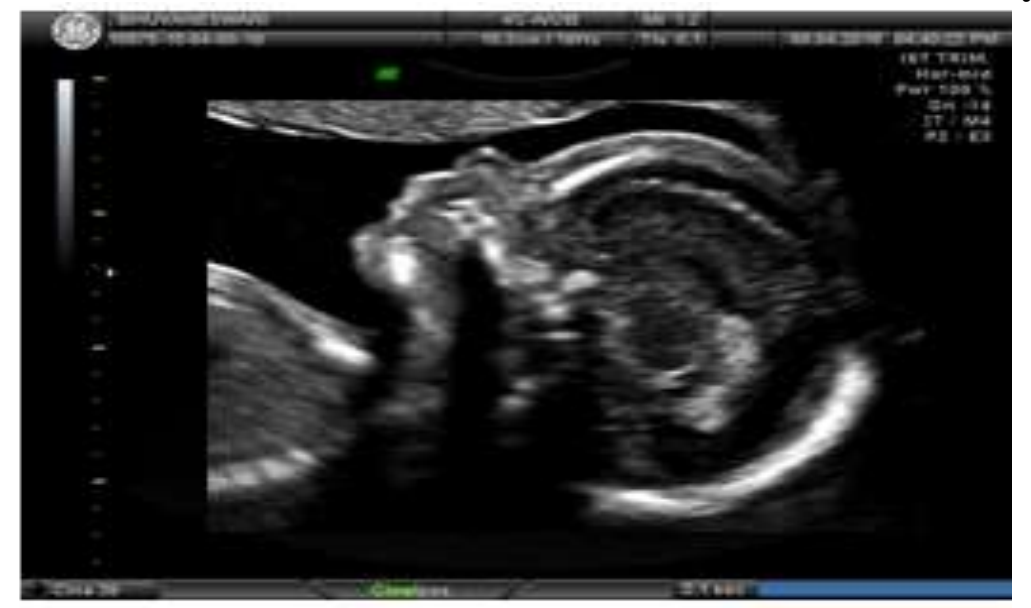

Fetus with absent nasal bone with normal nuchal translucency

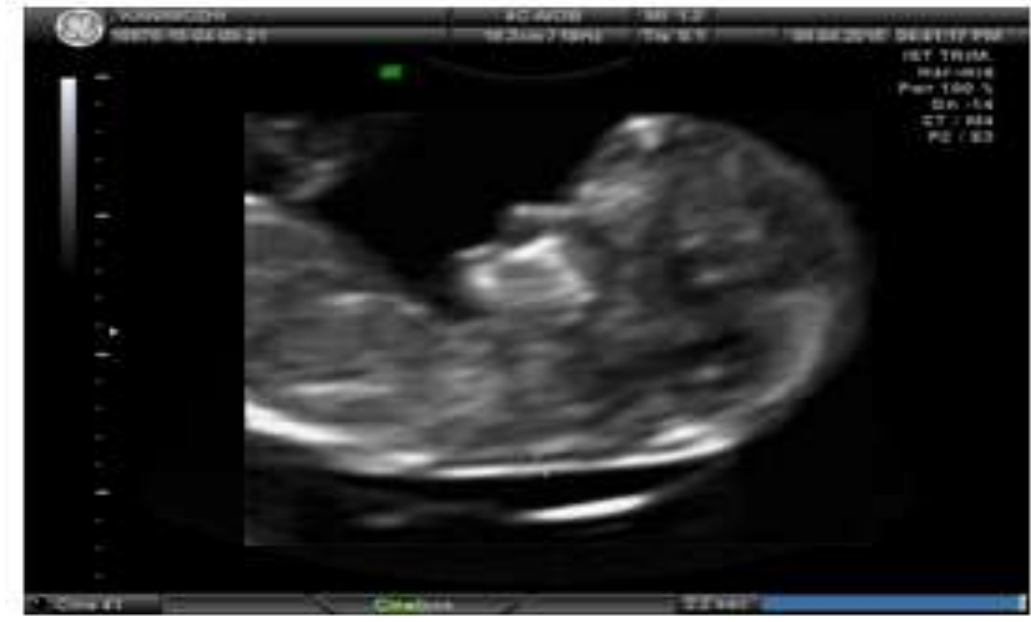




\section{Bibilography}

[1]. Association between first trimester absence of fetal nasal bone on ultrasound and Down syndrome by Otaño L ${ }^{1}$, Aiello H, Igarzábal L, , Gadow EC, Matayoshi T

[2]. First-trimester examination of fetal nasal bone in the Chinese population by Chen $\mathrm{M}^{1}$, Lee CP, Tang R, Ou CQ, Tang M,HChan B.

[3]. Ultrasonographic evaluation of fetal nasal bone in a low-risk population at 11-13 + 6 gestational weeks by Ramos-Corpas $\mathrm{D}^{1}$, Santiago JC, Montoya F

[4]. First-trimester nasal bone evaluation for aneuploidy in the general population by Malone FD ${ }^{1}$, Ball RH, Nyberg DA, Comstock CH, Saade G, Berkowitz RL, Dugoff L, Craigo SD, Carr SR, Wolfe HM, Tripp T, D'Alton ME; FASTER Research Consortium

[5]. Integrated ultrasound and biochemical screening for trisomy 21 using fetal nuchal translucency, absent fetal nasal bone, free betahCG and PAPP-A at 11 to 14 weeks.by Cicero S ${ }^{1}$, Bindra R, Rembouskos G, Spencer K, Nicolaides KH

[6]. first-trimester nuchal translucency, nasal bones, and trisomy 21 in selected and unselected populations by Prefumo $\mathrm{F}^{1}$, Sairam S, Bhide A, Thilaganathan B 7

[7]. To evaluate the feasibility and diagnostic accuracy of fetal nuchal translucency and nasal bone assessment at 11-14 weeks for screening of trisomy 21 at a single center by Monni G ${ }^{1}$, Zoppi MA, Ibba RM, Floris M, Manca F, Axiana C

[8]. Evaluation of absence or shortened nasal bone (Kanellopulos 2003, Otano 2002, Orlandi 2003) in prenatal screening

[9]. Nasal bone hypoplasia during the second trimester is associated with a high risk of Trisomy 21 (Bromley 2002, Cicero 2003, Odibo 2004, Guis Ville 1995), Cicero et al. 